\title{
The Gagliardo-Nirenberg-Sobolev Inequality for Non-Isotropic Riesz Potentials
}

\author{
Inan Cinar \\ Yuzuncu Yil University, Faculty of science, Department of Mathematics, 65080 VAN, Turkey.
}

Received: September 10, 2014 / Accepted: October 08, 2014 / Published: February 25, 2015.

Abstract: In this study, the inequality of Gagliardo-Nirenberg-Sobolev type are established for non-isotropic Generalized Riesz Potential depending on $\lambda$-distance.

Keywords: Non-isotropic distance, potentials

\section{Introduction}

In this paper, we establish analogues well known Gagliardo-Nirenberg-Sobolev inequality [2] for Riesz potentials with non-isotropic kernel depended on $\lambda$ - distance. Note that different problems for convolution type integrals with kernels depending on $\lambda$ - distance were considered in [1], [3-5].

Let $\lambda_{1}, \lambda_{2}, \ldots, \lambda_{n}$ be positive numbers with $|\lambda|=\lambda_{1}+\lambda_{2}+\ldots+\lambda_{n}$ and $\|x\|_{\lambda}=\left(\left|x_{1}\right|^{1 / \lambda_{1}}+\left|x_{2}\right|^{1 / \lambda_{2}}+\ldots+\left|x_{n}\right|^{1 / \lambda_{n}}\right)^{\frac{|\lambda|}{n}}, x \in \mathbb{R}^{n}$. The expression $\|x-y\|_{\lambda}, x, y \in \mathbb{R}^{n}$ is called the $\lambda-$ distance between the points $x$ and $y$.

Now we set

$$
\Lambda_{\alpha} f(x)=\int_{\mathbb{R}^{n}}\|x-y\|_{\lambda}^{\alpha-n} f(y) d y, 0<\alpha<n
$$

this integral is called non-isotropic $\lambda$ - Riesz potential.

Theorem 1. (Gagliardo-Nirenberg-Sobolev inequality). Assume $1 \leq p<q<n, \frac{1}{q}=\frac{1}{p}-\frac{\alpha}{n}$. There exists a constant $C$, depending only $p, q$ and $n$, such that

Corresponding author: Inan Cinar, Yuzuncu Yil University, Faculty of science, Department of Mathematics, 65080 VAN, Turkey. E-mail: inancinar@mynet.com.

$$
\left\|\Lambda_{\alpha} f\right\|_{L_{q}\left(\mathbb{R}^{n}\right)} \leq C\|D f\|_{L_{p}\left(\mathbb{R}^{n}\right)}
$$

for all $f \in C_{c}^{1}\left(\mathbb{R}^{n}\right)$.

Proof. It is obvious that,

$$
\begin{aligned}
\Lambda_{\alpha} f(x) & =\int_{\mathbb{R}^{n}} f(x-y) E_{1}(y) d y \\
& +\int_{\mathbb{R}^{n}} f(x-y) E_{\infty}(y) d y \\
& =I_{1}(x)+I_{2}(x)
\end{aligned}
$$

where for some positive $\mu$

$$
\begin{aligned}
& E_{1}(y)= \begin{cases}\|y\|_{\lambda}^{\alpha-n}, & \text { if }\|y\|_{\lambda} \leq \mu \\
0, & \text { if }\|y\|_{\lambda}>\mu\end{cases} \\
& E_{\infty}(y)= \begin{cases}0, & \text { if }\|y\|_{\lambda} \leq \mu \\
\|y\|_{\lambda}^{\alpha-n}, & \text { if }\|y\|_{\lambda}>\mu .\end{cases}
\end{aligned}
$$

Applying the $L_{p}$ norm we can see that

$$
\begin{aligned}
& \left(\int_{\mathbb{R}^{n}}\left|I_{1}(x)\right|^{p} d x\right)^{1 / p} \\
& =\left(\int_{\mathbb{R}^{n}}\left|\int_{\mathbb{R}^{n}} E_{1}(y) f(x-y) d y\right|^{p} d x\right)^{1 / p} \\
& \leq \int_{\mathbb{R}^{n}}\left|E_{1}(y)\right|\left(\int_{\mathbb{R}^{n}}|f(x-y)|^{p} d x\right)^{1 / p} d y \\
& \quad=\int_{\mathbb{R}^{n}}\left|E_{1}(y)\right|\left(\int_{\mathbb{R}^{n}}|f(t)|^{p} d t\right)^{1 / p} d y
\end{aligned}
$$




$$
\left\|I_{1}\right\|_{L_{p}} \leq C\left\|E_{1}(y)\right\|_{L_{1}}
$$

Note that the norm $\left\|E_{1}\right\|_{L_{1}}$ can be easy calculated. Namely, passing to spherical coordinates by tronsformation $x_{j}=\left(\gamma \theta_{j}\right)^{\lambda_{j}}, j=1,2, \ldots, n$, where $\theta_{j}$ are the coordinates of the point $\theta$ on unit sphere, we can see that Jacobian of this transformation is $\gamma^{2|\lambda-1|} \Omega(\theta)$ depend only on angles. Consequently, denoting $C_{1}=\int_{S^{n-1}} \Omega(\theta) d \theta$ where $S^{n-1}$ is the unite sphere in $\mathbb{R}^{n}$. We obtain

$$
\left\|E_{1}(y)\right\|_{L_{1}}=\int_{\|y\|_{\lambda} \leq \mu}\|y\|_{\lambda}^{\alpha-n} d y \leq C_{1} \mu^{2 \frac{|\lambda|}{n} \alpha} .
$$

Thus $I_{1}$ is finite. For $E_{\infty}(y)$ applying Hölder inequality

$$
\begin{aligned}
\left\|E_{\infty}(y)\right\|_{q} & =\left(\int_{\|y\|_{\lambda}>\mu}\|y\|_{\lambda}^{(\alpha-n) q} d y\right)^{1 / q} \\
& =C\left(\int_{\mu}^{\infty} \rho^{2 \frac{|\alpha|}{n}(\alpha-n) q} \rho^{2|\lambda|-1} d y\right)^{1 / q} \\
& =\left.C\left(\rho^{2 \frac{|\alpha|}{n}(\alpha-n) q+2|\lambda|}\right)^{\frac{1}{q}}\right|_{\mu} ^{\infty} .
\end{aligned}
$$

By hypothesis

$$
2 \frac{|\lambda|}{n}(\alpha-n)+2 \frac{|\lambda|}{q}=2|\lambda|\left(\frac{\alpha}{n}-\frac{1}{p}\right)<0 .
$$

Thus

$$
\left\|E_{\infty}\right\|_{q}<C \text {. }
$$

By using (1) and (2) inequalities,

$$
\left|\Lambda_{\alpha} f\right| \leq|f(t)| \text {. }
$$

Now first, assume $p=1 . f$ has compact support for each $i=1,2, \ldots, n, x \in R^{n}$, we have

$$
f(t)=\int_{-\infty}^{t_{i}} U_{t_{i}}\left(t_{1}, \ldots, t_{i-1}, y_{i}, t_{i+1}, \ldots, t_{n}\right) d y_{i}
$$

and so

$$
\begin{aligned}
& \left|\Lambda_{\alpha} f\right| \leq|f(t)| \leq \\
& \int_{-\infty}^{+\infty}\left|D f\left(t_{1}, \ldots, t_{i-1}, y_{i}, t_{i+1}, \ldots, t_{n}\right)\right| d y_{i} .
\end{aligned}
$$

Consequently,

$$
\begin{aligned}
& |f(t)|^{\frac{n}{n-\alpha}} \leq \\
& \prod_{i=1}^{n}\left(\int_{-\infty}^{+\infty}\left|D f\left(t_{1}, \ldots, t_{i-1}, y_{i}, t_{i+1}, \ldots, t_{n}\right)\right| d y_{i}\right)^{\frac{1}{n-\alpha}}
\end{aligned}
$$

integrate this inequality with respect to $t_{1}$

$$
\begin{aligned}
\int_{-\infty}^{+\infty}|f|^{\frac{n}{n-\alpha}} d t_{1} & \leq\left(\int_{-\infty}^{+\infty}|D f| d y_{1}\right)^{\frac{1}{n-\alpha}} \\
& \cdot\left(\prod_{i=2}^{n} \int_{-\infty}^{+\infty} \int_{-\infty}^{+\infty}|D f| d t_{1} d y_{i}\right)^{\frac{1}{n-\alpha}} .
\end{aligned}
$$

The last inequality resulting from general Hölder inequality.

We continue by integrating with respect to $t_{2}, t_{3}, \ldots, t_{n}$ eventually to find.

$$
\begin{gathered}
\int_{\mathbb{R}^{n}}\left|\Lambda_{\alpha} f\right|^{\frac{n}{n-\alpha}} d x \\
\leq \prod_{i=1}^{n}\left(\int_{-\infty}^{+\infty} \ldots \int_{-\infty}^{+\infty}|D f| d t_{1} \ldots d y_{i} \ldots d t_{n}\right)^{\frac{1}{n-\alpha}} \\
=\left(\int_{\mathbb{R}^{n}}|D f| d t\right)^{\frac{n}{n-\alpha}} .
\end{gathered}
$$

Consider now case that $1<p<n$ we apply estimate (3) to $v:=\left|\Lambda_{\alpha} f\right|^{\gamma}$ where $\gamma>1$ is to be selected. Then

$$
\begin{aligned}
& \left(\int_{\mathbb{R}^{n}}\left|\Lambda_{\alpha} f\right|^{\frac{\gamma n}{n-\alpha}} d x\right)^{\frac{n-\alpha}{n}} \\
& \leq C\left(\int_{\mathbb{R}^{n}}\left|\Lambda_{\alpha} f\right|^{(\alpha-1) \frac{p}{p-1}} d x\right)^{\frac{p-1}{p}}\left(\int_{\mathbb{R}^{n}}|D f|^{p} d x\right)^{\frac{1}{p}} \\
& =\left(\rho^{2 \frac{|\lambda|}{n}(\alpha-n) \frac{p}{p-1}+2|\lambda|}\right)^{\frac{p-1}{p}(\gamma-1)}\left(\int_{\mathbb{R}^{n}}|D f|^{p} d x\right)^{\frac{1}{p}}
\end{aligned}
$$

where

$$
2 \frac{|\lambda|}{n}(\alpha-n) \frac{p}{p-1}+2|\lambda|=2|\lambda|\left(\frac{\alpha}{n}-\frac{1}{p}\right)<0
$$

and $\gamma>1$. Finally,

$$
\left(\int_{\mathbb{R}^{n}}\left|\Lambda_{\alpha} f\right|^{q} d x\right)^{\frac{1}{q}} \leq C\left(\int_{\mathbb{R}^{n}}|D f|^{p} d x\right)^{\frac{1}{p}} .
$$


The proof is completed.

\section{References}

[1] İ. Çinar, The Hardy-Littlewood Sobolev inequality for non-isotropic Riesz Potentials, Turkish J. of Math., Vol. 21, Number 2 (1997), 153-157.

[2] C. Evans Lawrence, Partial Dif. Equations, Graduate Studies in Math., Vol. 19 (1998).
[3] İ. Çınar, On non-isotropic Riesz Potentials, Indian J. Pure Appl. Math., 28(9) (1997), p. 1197-1205.

[4] İ. Çinar, H. Duru, The Hardy-Littlewood Sobolev inequality of $(\beta, \gamma)$-distance Riesz Potentials, Appl. Mathematic and Computation, 153 (2004), 757-762.

[5] İ. Çınar, On non-isotropic $(\beta, \gamma)$-distance and generalized Riesz Potentials, International J. of Appl. Math., Vol 8, No:3 (2002), 285-293. 